\title{
Efficacy and Safety of Modified Qing-Ying Decoction for Atopic Dermatitis in Children: Study Protocol for a Randomized Controlled Trial
}

\section{Mei Kwan LAI}

Chinese University of Hong Kong

Chi Him SUM

Chinese University of Hong Kong

Connie YC KAM

Chinese University of Hong Kong

Tianhe SONG

Chinese University of Hong Kong https://orcid.org/0000-0002-2993-9594

\section{Pui Kuan CHEONG}

Chinese University of Hong Kong

\section{Kam Leung CHAN}

Chinese University of Hong Kong

\section{Steven KF LOO}

Chinese University of Hong Kong

Ellis KL HON

Chinese University of Hong Kong

Hongwei ZHANG

Chinese University of Hong Kong

Louis CW LO

Chinese University of Hong Kong

Jessica YL CHING

Chinese University of Hong Kong

Zhi-Xiu LIN ( $D$ linzx@cuhk.edu.hk)

The Chinese University of Hong Kong https://orcid.org/0000-0001-8635-0658

\section{Study protocol}

Keywords: Atopic dermatitis, modified Qing-Ying Decoction (mQYD), Chinese medicine, Randomized controlled trial, Gut microbiota

Posted Date: June 14th, 2021 
DOI: https://doi.org/10.21203/rs.3.rs-66124/v1

License: (c) (1) This work is licensed under a Creative Commons Attribution 4.0 International License. Read Full License 


\section{Abstract}

\section{Background}

Atopic Dermatitis (AD), also known as atopic eczema, is a chronic skin disease characterized by recurring episodes of itchiness with skin erythema and surface damages, such as dry skin, skin thickening and swelling. Long-term treatment with conventional medicine for subacute and moderate atopic eczema is not satisfactory. Evidence from randomized clinical trials suggests the effectiveness of Chinese medicine for the treatmement of AD. The purpose of this study is to explore the efficacy and safety of the modified Qing-Ying Decoction (mQYD) for the treatment of subacute and chronic AD in children through examining the clinical symptoms, quality of life, gut microbiome, and Chinese medicine (CM) body constitution.

Methods/design

This is a parallel, randomized, placebo-controlled, double-blind clinical trial with a 12-week treatment and a 4-week follow-up period. A total of 64 participants will be randomly allocated to the treatment group receiving oral mQYD granules or the placebo control group receiving oral placebo granules. Eczema Area and Severity Index (EASI) score will be the primary outcome. Secondary outcome measures include change in symptoms using the Patient-Oriented Eczema Measure (POEM), the Children's Dermatology Life Quality Index (CDLQI), and the use of concomitant medicines as well as the change in the gut microbiome and CM body constitution types after the 12-week treatment of the MQYD and 4-week followup compared to placebo control. Safety parameters include report of adverse events and pathology tests during the trial period.

\section{Discussion}

This is the first clinical trial of its kind to test the efficacy and gut microbiome of an innovative Chinese herbal formula modified from a famous ancient formula for the treatment of AD. The outcomes of this trial will provide clinical evidence on the effectiveness and safety of mQYD for the treatment of $A D$.

Trial registration

ClinicalTrials.gov, ID: NCT04419584. Registered on 28 July 2020.

\section{Background}

Atopic Dermatitis (AD) is a chronically relapsing skin disease characterized by recurring episodes of itchiness with erythema, dryness, thickening and swelling of the skin [1, 2]. AD skin lesions usually appear on the face, neck, back of the hands and feet, while itchiness and sleep loss are the most significant clinical symptoms. AD affects around $15 \%-30 \%$ of children and $2 \%-10 \%$ of adults worldwide $[3,4]$. In Hong Kong, the incidence rate of $A D$ was reported to be about $15-20 \%$ of the general population [5]. $A D$ lesions usually appear on the face, neck, back of the hands and feet. It is characterized by severe skin prutitus which inevitably leads to skin scratching [6]. Gerbens et al. conducted a systematic review 
related to the symptoms of AD with 295 articles included, and found that itchiness (98\% of reviewed articles) and sleep loss (61\% of reviewed articles) were the most common symptoms of AD [7]. The other symptoms also included skin dryness, oozing, erythema, scratching and peeling. According to a study by Wang et al. on the clinical features of $A D$ in China, it found that the mean age among the subjects with $\operatorname{AD}(n=682)$ was $28.8 \pm 20.1(p<0.001)$, and the median course of $A D$ was $5.3 \pm 6.9$ years, which was much longer than the other types of dermatitis (2.8 \pm 4.8 years) [8]. Besides, the severity of itchiness with bacterial infection in AD patients was more severe than the other types of dermatitis. The common involved sites of the body included fossa cubitalis, knees, neck, upper limbs and face. The lesions such as erythema, xerosis, papule, scratch and scale were also commonly presented in AD patient.

$A D$ has a profound negative impact on the quality of life for the patients and inflicts substantial financial burden to their families [3]. In the United States, the annual cost for the nation-wide mangement of AD ranged from US\$364 million to $\$ 3.8$ billion. In Australia, the annual personal cost was AU $\$ 330,818$ and 1,255 for managing mild, moderate and severe $A D$ respectively [9]. Other than the above direct cost, the indirect cost such as pain and absence from school is difficult to measure. Due to the discomfort and negative impact on patients' appearance, $A D$ often leads to psychological distress, including poor selfesteem, frustration, depression and social isolation [10]. AD is well-known for affecting the patients' sleep pattern and quality of life (6).

The causes of AD have not been fully understood [2]. Genetic factors and defects in the immune system such as decreased antimicrobial peptides and innate immune cells to the skin are implicated in the etiology of $A D$ [1]. In addition, it is also believed that the interaction between gut microbes and the intestinal immune system contributes to the occurrence of the skin disease $[11,12]$. The gut bacterial species of Faecalibacterium prausnitzii was associated with the impairment of the gut epithelial barrier, and contributed to the development of $A D$ [12].

Currently, there is no cure for AD. Topical application of corticosteroids and emollients is the mainstay of treatment for mild $A D$, while systemic therapies including oral steroids or immunosuppressive agents are used for refractory moderate or severe AD $[2,13]$. The use of systemic steroids is limited due to their systemic side effects, including growth suppression in children, osteoporosis, osteonecrosis and adrenal insufficiency [13]. Topical calcineurin inhibitors (TCls) suppress T-lymphocyte activation, but their use is limited because of application-site infections and a boxed warning for potential malignancy risk [10].

Chinese medicine (CM) is nowadays widely used for managing $A D$ in China and other East Asian countries. Several research groups worldwide have previously conducted clinical studies to evaluate the effectiveness of Chinese herbal medicines for the treatment of $A D$ in recent decades [11,12]. Findings from systematic reviews suggested that oral administration of a Chinese herbal medicine (CHM) formulation may improve health-related quality of life (HRQoL) of children with moderate or severe $A D$ [reference needed]. However, well-designed randomized controlled trials (RCTs) have been few and far between, and are badly needed to provide reliable clinical evidence to support the use of Chinese herbal 
medicine for the management of AD. Previous studies have demonstrated that the effect of Chinese herbal medicines on $A D$ may be related to their ability to modulate immune functions and antiinflammatory effect (14-16). Till now, few adverse effects related to Chinese herbal medicines for AD have been found [13,17]. A review conducted by Gu et al. [18] on the effectiveness of Chinese medicine for $A D$ found that only minor adverse events such as gastrointestinal upset were detected in a total of 2,306 participants receiving Chinese medicine intervention in 28 studies.

Among different Chinese herbal formulae for AD, Qing-Ying Decoction (QYD, Clear the Nutritive Level Decoction) is one of the most commonly used formulae, especially for $A D$ patients on the acute or subacute stage [19]. From CM perspective, $A D$ is mainly caused by the attack of pathogenic factors such as wind, dampness, and heat, and when these pathogenic factors lodge in the body surface, they cause skin erythema and surface damages. QYD has the function of dispelling pathogenic heat in the superficies and removing the toxicity, therefore it can relieve the AD symptoms such as erythema. QYD was a famous CM formula that first appeared in the "Itemized Differentiation on Epidemic Febrile Diseases" published in 1798, and is composed of Cornu Bubali (Shui-niu-jiao), Radix Rehmanniae (Sheng-di-huang), Radix Scrophulariae (Xuan-shen), Sinocalamus affinis (Zhu-ye-xin), Radix Ophiopogonis (Mai-dong), Radix Salviae Miltiorrhizae (Dan-shen), Radix Coptis chinensis (Huang-lian), Flos Lonicera japonica (Jīn-yin-hua) and Fructus Forsythia (Lian-qiao) [20]. However, the studies on QYD for $A D$ was sparse. Tsui and his colleagues published an article related to the commonly used $C M$ formulae for managing $A D$ and found that QYD was one of the commonly used formulae for treating $A D$ caused by heat, regardless internal or external heat. This formula was able to increase body immunity and exert anti-inflammatory effect, thus could manage AD effectively [21].

The present study aims to examine the therapeutic effect of modified Qing-Ying Decoction (mQYD) for the treatment of subacute and chronic AD. In CM perspective, pathogenic factors such as spleen deficiency, damp-heat accumulation, and lung heat congestion may contribute to the development of AD. The mQYD better suits the treatment need of the patients with subacute and chronic AD. Also, this study will use comprehensive outcome measures including physician assessments of disease severity, patient reported symptoms and quality of life outcomes in compliance with the CONSORT statement of Chinese herbal medicine [22]. Furthermore, this study will perform gut microbiome analysis to explore the relationship between the treatment response of $\mathrm{MQYD}$ and change of gut microbes, as well as evaluate its effect on $\mathrm{CM}$ body constitution types of participants using the Constitution in Chinese Medicine Questionnaire Hong Kong Version (CCMQ-HK) as one of the external anchor to reflect efficacy [23]. CCMQ-HK is the only valid patients reported outcomes that could reflect patient-centered care brought by Chinese medicine in Chinese population [23]. This randomized clinical trial (RCT) can in turn inform integrative medicine. Hence, a placebo-controlled, double-blind randomised clinical trial design with strong scientific rigor will be employed in this study, and it would be able to provide robust clinical evidence on the efficacy of mQYD. As AD is more prevalent in children in Hong Kong, this study will focus on children with $A D$. 
Besides, studies showed that the modes of delivery, infant feeding methods, and hygienic level of the house would influence the gut microbiota composition (GM, micros linked with human digestive tract) of infants, and the aberrance in GM composition has been hypothesized to be associated with the onset of AD. Studies have found that low quantities of Bifidobacterium, Bacteroide, Faecalibacterium and Lactobacillus paracasei, and/or with a high level of Enterobacteriaceae in infants, would increase the risk of developing $A D$ later in life. Intestinal bacterial colonization in early life is thought to help stimulate the protective antibody response, and activate the $T$ and $B$ cells proliferation and differentiation, therefore a lower risk in $A D$ could result if infants have diverse gut microbiota [24-27]. In order to have a clearer understanding of the relationship between $G M$ and $A D$ onset, a cohort study would be performed in this research. By collecting children's fecal samples, the richness of 16srDNA and the bio-diversity in each gut microbiome sample can be compared and investigated before and after taking mQYD [24-27].

\section{Objectives}

The study aims to evaluate the clinical efficacy and safety of the mQYD for the treatment of subacute and chronic $A D$ in children when compared to the placebo control through examining the clinical symptoms, quality of life, gut microbiome, and CM body constitution types after the 12-week treatment of the MQYD and 4-week follow-up.

\section{Methods/design}

\section{Trial design}

This is a parallel, randomized, placebo-controlled, double-blind clinical trial. Eligible subjects will be randomly allocated to the treatment group receiving oral MQYD granules or the placebo control group receiving oral placebo granules. Subjects will have 12-week of treatment, and then a 4-week follow-up.

\section{Outcomes}

\section{Primary outcome}

The primary outcome measure for this trial is the change of EASI score for atopic dermatitis at 12 weeks.

\section{Secondary outcomes}

The secondary outcomes of the trial include:

The change in scores of EASI at 6 and 16 weeks.

CDLQI and POEM and CM body constitution types at week 0, 12, and 16.

Gut microbiome will be assessed comparing week 0 and 12 . 
The time to and the number of subjects reaches flare (defined as major exacerbation disease increase > $50 \%$ of baseline EASI).

\section{Participants}

\section{Study population}

The subjects will be recruited from The Chinese University of Hong Kong Chinese Medicine Specialty Clinic cum Clinical Teaching and Research Centre and the Integrative Medical Centres of Hong Kong Institute of Integrative Medicine (HKIIM). Advertisements in posters which will be displayed at the clinics and on internet platforms such as Facebook, emails, and website of HKIIM will be conducted to promote community recruitment. Besides, we will publish articles in local newspapers and magazines as well as organize health promotion talks to augment the subject recruitment process. Potential participants with $A D$ who meet the eligibility criteria will be recruited. All participants will sign the informed consent form before the evaluation.

\section{Inclusion criteria}

The subjects who are diagnosed to have atopic dermatitis according to revised Hanifin and Rajka criteria suggested by the Guidelines of Care for the Management of Atopic Dermatitis [28, 29] will be assessed for eligibility. Subjects who meet all of the following criteria will be eligible for inclusion in the study:

1. Age of 4 to 17 (Chinese only),

2. With EASI $\geq 10$,

3. Subacute and chronic atopic dermatitis presenting with dry, scaly, erythematous papules and plaques, and

4. Provide written informed consent form (signed by one of their parents).

\section{Exclusion criteria}

Subjects with any one of the following conditions will be excluded from the study:

(1) History of allergy to Chinese medicines,

(2) AD with Yang deficiency in syndrome differentiation, such as pale looking, always feeling cold, fatigue, chronic diarrhea with loose stool, urinary disorders such as urinary difficulty, excessive urination or incontinence,

(3) Known overt bacterial infections in the skin,

(4) Known pregnancy,

(5) Known severe medical conditions, such as cardiovascular, liver or renal dysfunction or Diabetes Mellitus, 
(7) Having used oral corticosteroids, oral antibiotics, other immunosuppressive or any preparation of oral herbal medicines for the treatment of $A D$ in the past month,

(8) Having been diagnosed with scabies, allergic contact dermatitis, seborrheic dermatitis or psoriasis,

(9) Have taken anti-coagulant or anti-platelet drugs in the past month,

(10) Have taken probiotics, prebiotics in the last month, and

(11) Known history of glucose-6-phosphate dehydrogenase (G6PD) deficiency.

\section{Treatment schedule and follow up}

The subjects will come for a screening visit at week 0 (baseline), then at week $6 \pm 3$ days, week $12 \pm 3$ days and week $16 \pm 4$ days for assessments by Registered Chinese medicine practitioners (RCMPs) (Table 1). EASI, CDLQI, POEM and CM body constitution types will be assessed at different timepoints. They will receive oral herbal mQYD or placebo granules for 12 weeks, and then be followed up 4 weeks after the completion of treatment. RCMPs with at least 3 years' clinical experience will provide clinical consultation, prescribe and dispense MQYD or placebo at week 0 , and 6 to the subjects. A dermatologist will prescribe and dispense topical moisturizing cream and topical corticosteroid to the subjects for conventional and rescue therapy purposes during the study period, with the remaining unused cream being weighted at all subsequent visits. For each subject, photos covering the sites specified in EASI scoring will be taken at each visit. The lighting and background in the photographing process will be standardized and the photos will be normalized to improve the objectivity for the assessment. Study medication compliance and adverse events will also be assessed at each visit. Subjects will be instructed to send stool specimens to a microbiome laboratory at baseline and week 12 . A hotline will be provided for subjects to report any adverse event during office hours, and they will be recommended to attend Emergency Department at the nearest hospital beyond office hours if deemed necessary.

All subjects will be required to keep a daily record for monitoring their compliance with and side effects of the study treatment. They are also required to keep a record on the the frequency of using rescue treatment for AD. Also, they are advised to record all the other medication, including health food supplements such as probiotics taken during the period of study. Besides, they need to record three days of food diary before the day for taking faecal specimen for gut microbiome analysis as the food intake may affect the gut species and influence the gut microbiome analysis result. Furthermore, other than the mQYD prescribed by our CMP, the moisturizing cream and corticosteroid prescribed by our dermatologist, subjects are reminded not to take any other kinds of western or Chinese medicines or probiotics/prebiotics during the whole study period.

After completion of the study, all subjects can opt to join a special program for their AD at a discounted price. 


\section{Randomization and Blinding}

A computer program will be used to generate the random allocation list. The random allocations will be put into opaque envelops with sequential study numbers. Two sets of the envelope will be prepared, one set will be for randomization at the site and the other set stored in the investigator's office for emergency unblinding. Each subject will be assigned with a sequential study number and then the corresponding herbal granule or placebo will be prepared and allocated according to the random allocation sequence by a CM pharmacist. The dosages will be prepared according to the RCMP's instruction. In the study, the RCMP investigators, study subjects and outcome assessors will be kept blind of the medication allocation.

\section{Interventions}

The MQYD is prepared in the form of concentrated herbal granules based on the modified Qing-Ying Decoction with variable dosages according to subjects' weight.

Essentially, mQYD consists of Cornu Bubali (Shui-niu-jiao) $30 \mathrm{~g}$, Rehmanniae Radix (Sheng-di-huang) 25 g, Scrophulariae Radix (Xuan-shen) 12 g, Flos Lonicera japonica (Jin-yin-hua) 10 g, Fructus Forsythia (Lian-qiao) $10 \mathrm{~g}$, Cortex Dictamni (Bai-xian-pi) $10 \mathrm{~g}$, Periostracum Cicada (Chan-tui) $6 \mathrm{~g}$, Scutellariae Radix (Huang-qin) $10 \mathrm{~g}$, Rhizoma Alismatis (Ze-xie) $10 \mathrm{~g}$, Paeoniae Rubra Radix (Chi-shao) 12g, Cortex Phellodendri (Huang-bai) 10g, Semen Plantaginis (Che-qian-zi) 12g, and Adenophorae Radix (Nan-shashen) $12 \mathrm{~g}$.

One daily dose of MQYD herbal granules will be sealed into two packs, which will be dissolved in hot water and administered while it is lukewarm. Participants will take the medication twice daily, with one pack in the morning and the other in the evening after meals. Those children with the body weight $(\mathrm{BW})<$ $20 \mathrm{~kg}$ will take one-third of the total dose, between 20 to $30 \mathrm{~kg}$ of BW will take a half dose, between 30 to $40 \mathrm{~kg}$ of BW will take two-thirds of the total dose, and $>40 \mathrm{~kg}$ of BW will take normal dose.

The placebo granules, made of starch and caramel with similar appearance, smell and taste to the mQYD but contain no active constituents, will be taken by the patients in the placebo group in the same way as those in the treatment group. Both active treatment and placebo granules will be produced by a manufacturer with a Good Manufacturing Practice (GMP) certificate.

Since the subjects will be assessed by RCMPs under the guidance of Chinese medicine theory, the study medications are granules and the dosages will be adjustible, the study medications do not fall into the category of proprietary Chinese medicine, therefore, no certificate for Clinical Trial and Medicinal Test under Chinese Medicine Ordinance of Hong Kong (Cap 549) is needed.

All participants are allowed to use topical moisturizing cream prescribed by the dermatologist as conventional therapy, while topical mid-potency steroid cream, $0.1 \%$ mometasone furoate cream prescribed by the dermatologist can be used as rescue therapy if the condition does not reach flare. If a 
flare happens, the subject will reach an outcome, study treatment will be stopped and referral to a dermatologist for acute care will be confirmed by the dermatologist investigator.

\section{Possible side effects}

All subjects will be advised to report to the investigation team any adverse reactions during the treatment and follow-up period. Based on previous studies, the most common adverse reactions for Chinese herbal medicine treatment for $A D$ are gastrointestinal upset such as mild nausea and vomiting [30]. For moisturing cream, there are few RCTs reporting its adverse effect in great detail, though smarting, stinging, pruritus, erythema, folliculitis are the reported mild advese reaction [31]. Acneifrom eruption, atropic condition of skin, burning sensation, folliculitis, hypertrichosis, infection of skin and/or subcutaneous tissue, pruritus, rosacea, Cushing's syndrome, cataract and glaucoma have been reported as adverse effects in topical use of mometasone furoate [32], but clinically significant side effects of topical corticosteroids are thought to be rare, especially when in short-term use [33]. All adverse reaction will be recorded in the adverse drug reaction (ADR) form and the treatment will be suspended if severe adverse reaction occurs.

\section{Assessment Tools}

The following assessment tools will be used as the outcome measurements of the project:

1. EASI. EASI, which includes the assessment of 4 body regions (head and neck, trunk, upper limbs and lower limbs) by recording the percentage of affected skin in each region (area score) as well as the severity of $A D$ by measuring the intensity of redness, thickness, scratching and lichenification of these 4 regions will be used as the primary outcome. A dermatologist and a Research Assistant who is blind to the patient allocation and trained by a specialist in dermatology in western medicine will be responsible for conducting the EASI scoring.

2. Patient Oriented Eczema Measure (POEM). POEM will assess AD patients by evaluating the severity and duration of 7 symptoms (itch, sleep, bleeding, weeping/oozing, cracking, flaking, and dryness/roughness) experienced over the preceding week on a 5-point scale [34, 35].

3. Children's dermatology life quality index (CDLQI). CDLQI is used to measure the impact of skin problem on the quality of life to children under the age of 18 [36].

4. Gut microbiome analysis. The gut microbiota is the collection of microbial agents such as bacteria, viruses and fungi that inhabit in the gut. Fecal samples will be collected before and after the treatment. Bacterial microbiome sequencing is performed using $16 \mathrm{~S}$ ribosomal RNA pyrosequencing. Bioinformatics analysis will be performed by commercial microbiome laboratory.

5. Constitution of Chinese Medicine Questionnaire (CCMQ) [37-40]. This questionnaire consists of 60 items classifying individual into one of the nine constitutions: 8 items for gentleness (ping-he), 8 items for qi-deficiency (qi-xu), 7 items for yang-deficiency (yang-xu), 8 items for yin-deficiency (yin-xu), 8 items 
for phlegm-dampness (tan-shi), 6 items for damp-heat (shi-re), 7 items for blood-stasis (xue-yu), 7 items for qi-depression (qi-yu) and 7 items for special diathesis (te-bing) [23, 41, 42]. Based on TCM theory, the coexistence of body constitution (BC) is possible [23]. The Hong Kong version CCMQ (CCMQ-HK) used in this study has been validated in Hong Kong population with Chinese norm anchored with SF-12v2 HK norm [23]. The construct validity, reliability, sensitivity, and responsiveness were satisfactory [23].

\section{Sample Size}

The primary study objective is to compare the mean EASI between treatment and control group. Based on the previous study, we presume the standard deviation of EASI is 8.5. To detect the minimal clinically important difference of 6.6 in EASI [43] between the two groups, 27 subjects in each arm are needed with $80 \%$ power at a $5 \%$ two tailed significance level. To take account of a predicted maximum dropout rate of $20 \%$, we hence need 32 participants in each group, i.e. 64 participants in total for the project.

\section{Statistical analysis}

All analyses will be conducted according to the intention-to-treat principle. Descriptive statistics will be computed for each of the analyzed variables. The primary efficacy analysis will be done by comparing the two groups with respect to EASI at 12 weeks using analysis of covariance (ANCOVA). Supplementary analyses will include a complete-case analysis and a linear mixed model analysis of all available data. Repeated measures ANCOVA will be used to test for group differences in the secondary outcomes, with adjustment for relevant baseline covariates. All statistical tests will be two-sided, and $p<0.05$ is considered statistically significant. The statistical software of SPSS 22.0 will be used for analysis.

Adverse events will be categorized, and the percentage of those experiencing some adverse events and serious adverse events will be documented. Chi-square tests will be performed to examine differences in the proportion of total and categories of adverse events.

\section{Ethics consideration}

This study will comply with the Declaration of Helsinki and ICH-GCP (IVH-E6). Ethics approval has been obtained from the Joint Chinese University of Hong Kong - New Territories East Cluster Clinical Research Ethics Committee (Ref. No.: 2019.142). Participants and their parents will be ensured of the confidentiality and the clinical data-maintained anonymity. Consent forms should be signed by the subjects' parents or the subjects themselves (over 16 years of age) before the study. All information will be encrypted and only the involved investigators can have access. Password is required to access the data. Participants are free to withdraw at any time without giving a reason or punishment. The personal data of the subjects will only be kept for 7 years and will be destroyed afterwards. Clinical trial insurance will be purchased according to the university policy.

Table 1 Study Schedule 


\begin{tabular}{|c|c|c|c|c|}
\hline & $\begin{array}{l}\text { Week } 0 \text { (Baseline for } \\
\text { Randomization) }\end{array}$ & $\begin{array}{l}\text { Week } \\
6 \\
+/-3 \\
\text { days) }\end{array}$ & $\begin{array}{l}\text { Week } \\
12 \\
+/-3 \\
\text { days) }\end{array}$ & $\begin{array}{l}\text { Week } \\
16 \\
(+/-4 \\
\text { days) }\end{array}$ \\
\hline Informed Consent & $\mathrm{X}$ & & & \\
\hline $\begin{array}{l}{ }^{1} \text { Western medical consultation and } \\
\text { assessment }\end{array}$ & $\mathrm{X}$ & $X$ & $x$ & \\
\hline Chinese medical consultation and assessment & $\mathrm{X}$ & $x$ & $x$ & $\mathrm{x}$ \\
\hline Medical history & $x$ & & & \\
\hline Concomitant drug History & $\mathrm{X}$ & $\mathrm{x}$ & $\mathrm{X}$ & $\mathrm{x}$ \\
\hline EASI & $\mathrm{X}$ & $\mathrm{X}$ & $\mathrm{X}$ & $\mathrm{x}$ \\
\hline CDLQI, POEM and CCMQ & $x$ & & $\mathrm{X}$ & $\mathrm{x}$ \\
\hline${ }^{2} \mathrm{AE} / \mathrm{SAE}$ assessment & & $\mathrm{X}$ & $X$ & $\mathrm{X}$ \\
\hline $\begin{array}{l}\text { Subjects' daily record for study medication } \\
\text { compliance and rescue therapy }\end{array}$ & $\mathrm{X}$ & $\mathrm{X}$ & $\mathrm{X}$ & \\
\hline Stool collection & $x$ & & $\mathrm{x}$ & \\
\hline
\end{tabular}

${ }^{1}$ Western medical consultation and assessment: ${ }^{1}$ Western medical consultation at baseline can be skipped if the subject is diagnosed before randomization

${ }^{2} \mathrm{AE} / \mathrm{SAE}$ assessment: subjects will be referred to see Dermatologist investigators whenever needed.

\section{Discussion}

$A D$, a very common skin disease that mostly affects children, is characterized by redness of the skin, scaling, swelling, accentuation of the hair follicles and lichenification that result from chronic scratching owing to severe itchiness. $A D$ has a profound negative impact on the quality of life for the patients and inflicts substantial financial burden to their families.

Currently, there is no cure for AD. CM is nowadays widely used for managing AD in many East Asian countries and provides a potential alternative to conventional medicine in managing AD. This study aims to evaluate the clinical efficacy and safety of the modified Qing-Ying Decoction for the treatment of subacute and chronic AD using a double-blind, randomized, placebo-controlled design. Besides the EASI to be used as the primary outcome measure, the outcome assessments also include examination of clinical symptoms, quality of life, gut microbiome, and CM body constitution.

This is the first clinical trial of its kind to test the efficacy and the influence on gut microbiome of an innovative Chinese herbal formula modified from a famous ancient formula for the treatment of $A D$. The 
formula, if proven to be efficacious, will be used for further product development into an effective herbbased therapeutic modality that specifically targets children with AD. The product development as a result of this project would certainly provide an evidence-based Chinese herbal treatment for this distressing skin disease. Also, the evaluation of gut microbiome will help us to better understand the role of the gut microbiome in disease development and regression. Moreover, the results would be published in an academic journal, and utilized for a press conference to disseminate the research finding to a wider audience.

\section{Trial status}

This protocol is version 4 from 15 April 2020. Important protocol amendments will directly be communicated to all investigators, sponsors, registries, and committees. This trial is currently ongoing. The study commenced on 1 June 2020, and the anticipated end date of the study is 31 May 2022.

\section{Abbreviations}

AD: Atopic Dermatitis; ADR: Adverse drug reaction; ANCOVA: Analysis of covariance; BC: Body constitution; BW: Body weight; CCMQ-HK: Constitution in Chinese Medicine Questionnaire-Hong Kong Version; CDLQI: Children's Dermatology Life Quality Index; CHM: Chinese herbal medicine; CM: Chinese medicine; CMPs: Chinese medicine practitioners; EASI: Eczema Area and Severity Index; G6PD: Glucose-6phosphate dehydrogenase; GM: Gut microbiota; GMP: Good Manufacturing Practice; HKIIM: Hong Kong Institute of Integrative Medicine; HRQoL: Health-related quality of life; ICH-GCP: International Conference on Harmonisation-Good Clinical Practice; mQYD: Modified Qing-Ying Decoction; POEM: Patient Oriented Eczema Measure; QYD: Qing-Ying Decoction; RCT: Randomized controlled trial; RCMPs: Registered Chinese medicine practitioners; TCLs: Topical calcineurin inhibitors; TCM: Traditional Chinese Medicine.

\section{Declarations}

\section{Acknowledgement}

We thank Dr. Barbara Kwok for her ardent support to this project.

\section{Authors' contributions}

ZXL is the Principal Investigator of this trial. ZXL and JC conceived the study. MKL, CHS, CYCK, PKC, THS drafted the protocol. ZXL and JC revised the protocol. SL, KLC and EH contributed to patient recruitment and assessment. MKL, CHS and HWZ performed CM consultations. THS, LCWL and KLC were responsible for site-coordination. All authors read and approved the final manuscript.

\section{Funding}


The project is funded by Dr. Barbara Kwok IM Research Fund at the Hong Kong Institute of Integrative Medicine, CUHK. The funder had no role in the design, patient recruitment, data collection and interpretation of the study.

\section{Availability of data and materials}

The datasets used or analysed during the current study will be available from the corresponding author upon reasonable request.

\section{Ethics approval and consent to participate}

The study protocol was approved by The Joint Chinese University of Hong Kong - New Territories East Cluster Clinical Research Ethics Committee (Ref. No.: 2019.142). Informed consent will be obtained from all study participants.

\section{Consent for publication}

Not applicable

\section{Competing interests}

The authors declared that they have no competing interests.

\section{References}

1. Mayba JN, Gooderham MJ. Review of atopic dermatitis and topical therapies. J Cutan Med Surg. 2017;21(3):227-36.

2. Thomsen SF. Atopic dermatitis: natural history, diagnosis, and treatment. Int Sch Res Notices. 2014; 2014:354250.

3. DaVeiga SP, editor Epidemiology of atopic dermatitis: a review. Allergy Asthma Proc. 2012;33(3):22734.

4. Mansouri Y, Guttman-Yassky E. Immune pathways in atopic dermatitis, and definition of biomarkers through broad and targeted therapeutics. J Clin Med. 2015;4(5):858-73.

5. The Hong Kong Allergy Association. Eczema 2014. Available at: http://www.allergyhk.org/? page_id=317.

6. Jadotte YT, Santer M, Vakirlis E, et al. Complementary and alternative medicine treatments for atopic eczema. Cochrane Database Syst Rev. 2017(9): CD010938.

7. Gerbens LAA, Chalmers JR, Rogers NK, Nankervis H, Spuls PI. Reporting of Symptoms in Randomized Controlled Trials of Atopic Eczema Treatments: A Systematic Review. Br J Dermatol. 2016;175(4):678-86. 
1. Wang X, Li LF, Zhao DY, Shen YW. Prevalence and clinical features of atopic dermatitis in China. Biomed Res Int. 2016;2016:2568301.

2. Su JC, Kemp AS, Varigos GA, Nolan TM. Atopic eczema: its impact on the family and financial cost. Arch Dis Child. 1997;76(2):159-62.

3. Mancini AJ, Kaulback K, Chamlin SL. The socioeconomic impact of atopic dermatitis in the United States: a systematic review. Pediatr Dermatol. 2008;25(1):1-6.

4. Lee MJ, Kang MJ, Lee SY, Lee E, Kim K, Won S, et al. Perturbations of gut microbiome genes in infants with atopic dermatitis according to feeding type. J Allergy Clin Immunol. 2018;141(4):1310-9.

5. Song H, Yoo Y, Hwang J, Na YC, Kim HS. Faecalibacterium prausnitzii subspecies-level dysbiosis in the human gut microbiome underlying atopic dermatitis. J Allergy Clin Immunol. 2016;137(3):852-60.

6. Sherry HY, Drucker AM, Lebwohl M, Silverberg JI. A systematic review of the safety and efficacy of systemic corticosteroids in atopic dermatitis. J Am Acad Dermatol. 2018;78(4):733-40. e11.

7. Latchman Y, Banerjee P, Poulter L, Rustin M, Brostoff J. Association of immunological changes with clinical efficacy in atopic eczema patients treated with traditional Chinese herbal therapy (Zemaphyte®). Int Arch Allergy Immunol. 1996;109(3):243-9.

8. Lee J, Jung E, Park B, Jung K, Park J, Kim K, et al. Evaluation of the anti-inflammatory and atopic dermatitis-mitigating effects of BSASM, a multicompound preparation. J Ethnopharmacol. 2005;96(1-2):211-9.

9. Xu XJ, Banerjee P, Rustin M, Poulter L. Modulation by Chinese herbal therapy of immune mechanisms in the skin of patients with atopic eczema. Br J Dermatol. 1997;136(1):54-9.

10. Nagle TM, Torres SM, Horne KL, Grover R, Stevens MT. A randomized, double-blind, placebocontrolled trial to investigate the efficacy and safety of a Chinese herbal product (P07P) for the treatment of canine atopic dermatitis. Vet Dermatol. 2001;12(5):265-74.

11. Gu SX, Zhang AL, Coyle ME, Chen D, Xue CC. Chinese herbal medicine for atopic eczema: an overview of clinical evidence. J Dermatolog Treat. 2017;28(3):246-50.

12. Chen XY, Wu CY. Wu Chengyan's experience in treating eczema. Chin J Basic Med Tradit Chin Med. 2018;24(5):693-5.

13. Wang Q, Zhang ZQ, Qi WB. Research progress of Qing-Ying Decoction in clinical application. Heilongjiang Chin Med. 2012;41(6):57-8.

14. Sui HF, Liu B, Li C, Gao LX. Modern clinical application of Qing-Ying Decoction. Neimenggu Zhongyiyao. 2016;5:147-8.

15. Cheng CW, Wu TX, Shang HC, Li YP, Altman DG, Moher D, et al. CONSORT Extension for Chinese Herbal Medicine Formulas 2017: Recommendations, Explanation, and Elaboration. Ann Intern Med. 2017;167(2):112-21.

16. Wong W, Lam CLK, Wong VT, Yang ZM, Ziea ETC, Kwan AKL. Validation of the constitution in Chinese medicine questionnaire: does the traditional Chinese medicine concept of body constitution exist? Evid Based Complement Alternat Med. 2013;2013:1-14. 
17. Kim JE, Kim HS. Microbiome of the skin and gut in atopic dermatitis (AD): understanding the pathophysiology and finding novel management strategies. J Clin Med. 2019;8(4):444.

18. Petersen E, Skov L, Thyssen J, Jensen P. Role of the gut microbiota in atopic dermatitis: a systematic review. Acta Derm Venereol. 2019;99(1-2):5-11.

19. Reddel S, Del Chierico F, Quagliariello A, Giancristoforo S, Vernocchi P, Russo A, et al. Gut microbiota profile in children affected by atopic dermatitis and evaluation of intestinal persistence of a probiotic mixture. Sci Rep. 2019;9(1):1-10.

20. Liu SP, Zhang ZX, Chen ZQ. Talking about intestinal microflora and skin diseases. Mon J ClinMed. 2019;84(2):554-8.

21. Eichenfield LF, Hanifin JM, Luger TA, Stevens SR, Pride HB. Consensus conference on pediatric atopic dermatitis. J Am Acad Dermatol. 2003;49(6):1088-95.

22. Eichenfield LF, Tom WL, Chamlin SL, Feldman SR, Hanifin JM, Simpson EL, et al. Guidelines of care for the management of atopic dermatitis: section 1. Diagnosis and assessment of atopic dermatitis. J Am Acad Dermatol. 2014;70(2):338-51.

23. Gu S, Yang AW, Xue CC, Li CG, Pang C, Zhang W, et al. Chinese herbal medicine for atopic eczema. Cochrane Database Syst Rev. 2013;10(9):CD008642.

24. van Zuuren EJ, Fedorowicz Z, Christensen R, Lavrijsen APM, Arents BWM. Emollients and moisturisers for eczema. Cochrane Database Syst Rev. 2017;2017(2):CD012119.

25. IBM Micromedex ${ }^{\circledR}$ DRUGDEX ${ }^{\circledR}$ (electronic version). IBM Watson Health, Greenwood Village, Colorado, USA. Available at: https://www-micromedexsolutions-com.libproxy.uthscsa.edu/(cited: Mar 25, 2020).

26. Berth-Jones J. Topical Therapy. Rook's Textbook of Dermatology. 1, Eighth Edition.. 2010. 73.1-52.

27. Charman C, Venn A, Ravenscroft J, Williams H. Translating Patient-Oriented Eczema Measure (POEM) scores into clinical practice by suggesting severity strata derived using anchor-based methods. Br J Dermatol. 2013;169(6):1326-32.

28. Schmitt J, Spuls P, Boers M, Thomas K, Chalmers J, Roekevisch E, et al. Towards global consensus on outcome measures for atopic eczema research: results of the HOME II meeting. Allergy. 2012;67(9):1111-7.

29. Lewis-Jones M, Finlay A. The Children's Dermatology Life Quality Index (CDLQI): initial validation and practical use. Br J Dermatol. 1995;132(6):942-9.

30. Wang Q. Classification and diagnosis basis of nine basic constitutions in Chinese medicine. J Beijing Univ Tradit Chin Med. 2005;28(4):1-8.

31. Wang Q. Compiling and application of constitution in Chinese medicine questionnaire China J Tradit Chin Med Pharmacy. 2006;Supplement:54-7.

32. Wang Q, Zhu YB, Xue HS, Li S. Primary compiling of constitution in Chinese medicine questionnaire. Chin J Clin Rehabil. 2006;10(3):12-4. 
33. Zhu YB, Wang Q, Xue HS, Orikasa Q. Preliminary assessment on performance of constitution in Chinese medicine questionnaire.Chin J Clin Rehabil. 2006;10(3):15-7.

34. Ren XJ, Wang Q. Discussion on application of tradicitonal Chinese medicine classification standards of health in personal health management. Chin Health Serv Manag. 2007;23(9):580-1.

35. Wang J, Li YS, Ni C, Zhang HM, Li LR, Wang Q. Cognition research and constitutional classification in Chinese medicine. Am J Chin Med. 2011;39(4):651-60.

36. Schram M, Spuls PI, Leeflang M, Lindeboom R, Bos J, Schmitt J. EASI,(objective) SCORAD and POEM for atopic eczema: responsiveness and minimal clinically important difference. Allergy. 2012;67(1):99-106.

\section{Supplementary Files}

This is a list of supplementary files associated with this preprint. Click to download.

- SPIRITFillablechecklistAD.pdf 